\title{
DETECTION OF CYST IN HUMAN BRAIN USING SCAN IMAGES
}

\author{
Debnath Bhattacharyya ${ }^{1}$ and N. Thirupathi Rao ${ }^{1}$ \\ ${ }^{1}$ Department of Computer Science and Engineering, \\ Vignan's Institute of Information Technology, \\ Visakhapatnam- 530049, AP, India \\ nakkathiru@gmail.com, debnathb@gmail.com
}

\begin{abstract}
A Colloid Cyst is a cyst containing gelatinous material in the brain. It originates from the roof of the ventricle, which contains the cerebrospinal fluid. A Colloid Cyst represents 0.5\%-2\% of intracranial tumours. Circulation of the cerebrospinal fluid (CSF) can be blocked for better health. Intracranial pressure also increased due to the building up of CSF causing brain damage and finally sudden death. In the current paper, an algorithm with use of Median Filter to Noise Reduction on object boundaries in an image that obtains through Sobel Edge Detector was proposed. Our proposed system consists of three phases: PreProcessing, Segmentation and Feature Extraction. This methodology helps us to accurately detect and simplify the process of detecting the presence of a colloid cyst in the brain from MRI or CT scanned images.
\end{abstract}

Keywords - Colloid Cyst detection, Threshold Segmentation, Median Filtering, Gaussian high-pass filtering, Region of Interest.

\section{INTRODUCTION}

In our human body, the brain is the central organ of the central nervous system that coordinates and controls the activities of other organs in our body. A group of cells in the brain called as cysts, all these cells clustered together to form a cyst that contains fluid or semi-solid material such as blood, cerebrospinal fluid, tissue or tumour cells[1, 2]. Cysts are generally benign but found in parts of the brain. These cysts are harmful, and they restrict the basic functioning of the human brain. There are different types of Cysts found in the human brain. Some of them are Colloid Cyst, Arachnoid Cyst, Dermoid Cyst, Pineal Cyst, Epidermoid Cyst and Tumor-associated Cyst. Symptoms of cysts have differences depending upon its size, location and type of the cyst.

In this paper, we focus on the automatic detection of Colloid Cyst in Brain from MRI or CT scanned images. Colloid cysts are known to be formed during the embryonic formation of the Central Nervous System. It contains a thick, gelatinous substance called colloid which came from the Greek word Kollodes (Kolla meaning glue and eidos meaning appearance). Apart from the colloid filling, the cyst may contain blood, minerals or cholesterol crystals[3]. Colloid Cysts found in the centre of the brain that holds spinal fluid, or in the lining of the third ventricle. Cysts in this location block the foramina of Monro causing obstructive hydrocephalus that increases pressure in the brain. Familiar symptoms are a severe headache, nausea, vomiting, seizures, vertigo, memory loss, insomnia, gait disorder, drop attack and many more. Mortality rate due to Colloid cyst has been between $58 \%$ and $77 \%$ [2, 3, 4]. Its size may vary from 3 to $40 \mathrm{~mm}$. Since even

Received: November 23, 2018

Reviewed: January 28, 2019

Accepted: February 1, 2019

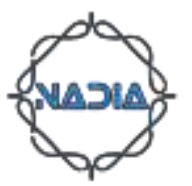


small Colloid cyst can cause sudden death; it is vital to identify or detect the cyst at an early stage [5].

Now a day's, Medical Image Processing has become an essential feature in the fields of Bio-Medical research. Imaging Technology like Magnetic Resonance Imaging, CT scanner, and digital mammography provides a detailed or third dimension view of the body [6]. The digital images acquired from these imaging technologies can be enhanced and analysed by MATLAB and Image Processing Toolbox for easier diagnosis. Image Processing Techniques reduces the complicated manual tasks of the radiologists to identify any abnormalities in the brain; it saves time and is cost effective. It involves preprocessing of the digital images by passing them through different types of filters to reduce noise and improve the quality of the image. It also includes emphasising on the region of interest and automating the process of segmentation of Cysts to extract complex information. Morphological operations can be used to remove imperfections from these images depending on the relative ordering of the pixels [7]. We have proposed here an algorithm that uses many advanced image processing techniques to automatically detect Colloid cyst of all sizes from the digital images. To test our algorithm, we have used 100 neuroimages that contained Colloid Cyst and 50 neuroimages where there was the absence of any abnormalities or contained cysts but not Colloid Cyst. The result demonstrated that the simple algorithm was efficient enough to detect Colloid Cyst of any size automatically [8]. According to the region of interest or location, the algorithm was successful to recognise if there was an absence of cyst or the cyst present was the colloid cyst or not.

\section{LITERATURE REVIEW}

Al-Shariah AM et al., had discussed in detail about the various types of cysts can present in the human brain and various problems that patients may face due to the presence of these cysts. They further discussed the various set of cases for identifying and confirming the presence of cysts with examples [1].On a different note Alexander C. Mamourian et al., presented different cases and tried to prove that Colloid Cyst is sometimes more perceptible or noticeable on Computed Tomography Images than Magnetic Resonance Images. They stated cases where Colloid Cyst had found without any association with hydrocephalus and 55 cases where the cause of sudden death was due to Colloid Cyst in Brain ranging from 1 to $8 \mathrm{~cm}$. They concluded that "Ventricular size is not a reliable predictor of the outcome"[2].

Jeremy P. Morgan et al., had discussed a various set of cysts to be available for patients and which discussed in detail with four case studies. The case studies had presented in detail with the results they had got for identifying the cysts in brain images [3]. Karishma Sheikh et al., proposed a system that used pixel to pixel comparison, grey scale and K-means segmentation algorithm to detect a tumour from MRI images [4]. They used clustering to differentiate between affected and unaffected cells. EdEdilyMohd et al., localised Brain Tumor in Magnetic Resonance Imaging by applying edge detection method, modified histogram clustering and morphological operations [5]. They used a method where histogram values plotted, and the threshold value was kept fixed based on the pixels and grey level value in the images. Tumour part was extracted by using morphological operations and by specifying the Region of Interest.

Vrushali D. Dharmale and P.A. Tijare used Canny Edge detection and segmentation method for Cyst detection from MRI brain images and mentioned the accuracy rate to be $100 \%$ [6]. Lalit P. Bhaiya et al., classified abnormalities in Magnetic Resonance Brain images by developing a hybrid model that combined advantages of both Artificial Neural networks and Fuzzy Logic [7]. In this system, textural features were extracted using principal component analysis (PCA) technique. The extracted features were then used to train the neuro-fuzzy classifier. The Adaptive Network-based Fuzzy interference system 
(ANFIS) tested for classification of different Brain MRI samples. Results illustrated that the model was useful enough regarding classification accuracy and convergence rate.

Mashal Tariq et al., presented a system that focused on the early detection of a tumour [8]. In this paper, Noise Reduction made by the use of Median Filter, information about the object boundaries in an image obtained through Sobel Edge Detector. The algorithm proposed that several morphological operations along with morphological reconstruction could accurately segment out Solid cum Cystic Tumor from T1 and T2 images.

Shreetam Behera et al., have done a "Comparative Analysis on Edge Detection of Colloid Cyst"[9]. Based on simple mathematical morphology they provided an edge detection method to detect a Colloid Cyst in Brain. Sameer S Shaktawat, Walid D Salman, Zuhair Tiwaj and Abdul Al-Dawoud [10] wrote an article on "Unexpected death after a headache due to a colloid cyst of the third ventricle". What they presented a case of a 17-year-old female who had mild headaches for two years died suddenly, and the findings in the post-mortem report concluded that death was due to Colloid cyst of $1 \mathrm{~cm}$.

\section{PROPOSED METHODOLOGY}

In the current section, we proposed an algorithm with use of Median Filter to Noise Reduction on object boundaries in an image that obtains through Edge Detector. In this algorithm, several operations also are considered.

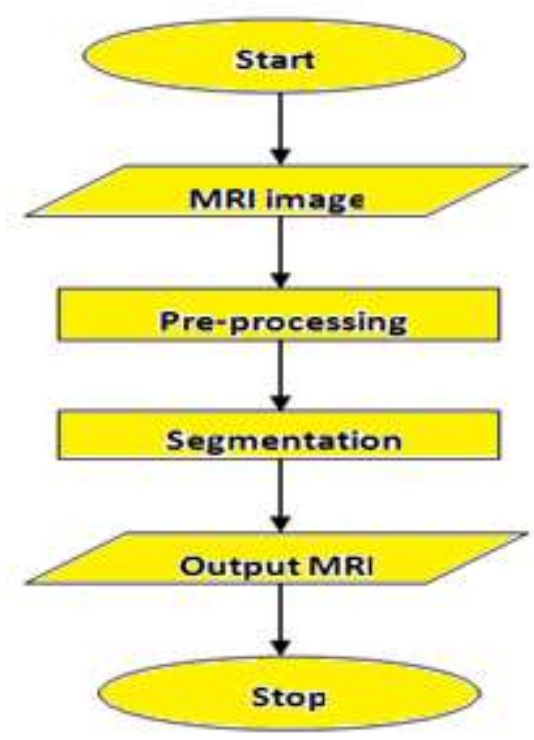

Fig. 1 Flowchart Model of the proposed method

The proposed System has three phases:

1. Pre-processing (Filtering, Contrast enhancement)

2. Segmentation and

3. Feature extraction.

In the first phase artefact and noise have been removed from the image and then the median filter has been applied to the resulting image. In the second phase, the desired portion that is colloid cyst had segmented. In the third phase represents the statistical features of the Colloid Cysts. A novel method had developed in this project which contains a self-constructed a practical algorithm for automated cysts detection and feature extraction based on MRI or CT images. 


\subsection{THE ALGORITHM TO DETECT COLLOID CYST}

Step 1: Start

Step 2: Read Input MRI/CT scanned Brain image

Step 3: Convert the input image into Grayscale image

Step 4: Apply Median filter on the Grayscale image to remove noise

Step 5: Pass the filtered image through Gaussian high pass filter

Step 6: Implement multilevel Thresholding on the filtered image

Step 7: Implement Segmentation using a threshold value

Step 8: Define the region of interested \&crop that portion

Step 9: If a cyst present in that location

Step 9.1: Trace the Region boundaries and plot the shape and part trace the region boundaries and plot the shape and location of the cyst in the

actual image.

Step9.1.1: $\quad$ Display the actual image with colloid cyst superimposed with red colour boundary.

Step 9.1.2: Calculate the size of the colloid cyst in $\mathrm{mm}^{2}$

Step9.1.3: Display a message box with the message box with "colloid cyst detected moreover, the approximate size of the colloid cyst is $\mathrm{mm}^{2}$ " else Step 9.2: Display actual image with the message "No colloid cyst present." Step 10: Stop

The proposed algorithm takes a minimal amount of time to detect the presence or absence of a Colloid Cyst, irrespective of the size of the image. Average time taken is between 10sec to $12 \mathrm{sec}$, and the accuracy rate is $98 \%$. It can detect tiny size Colloid Cyst as well. It extracts and marks the exact shape of the cyst, therefore making the calculation for the size of the Colloid Cyst almost accurate.

\subsection{IMPLEMENTATION OF ALGORITHM: CONVERSION OF INPUT IMAGE AND GRAY SCALE IMAGE}

We have taken Magnetic Resonance and Computed Tomography (MRI or CT scan) images of Brain as Input Image. By using the MATLAB functions, the input image can be converted into the grayscale image by using various functions. The input image may be of the RGB form and to process the further to convert into black and white images. This conversion process can be observed in the following images as follows,

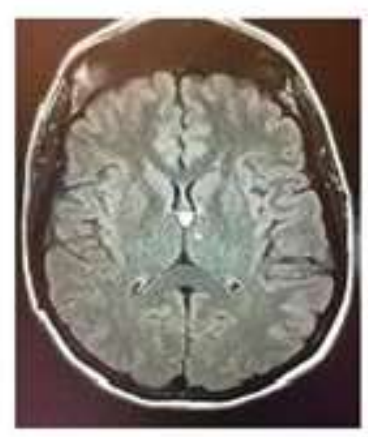

Fig. 2 24-bit image

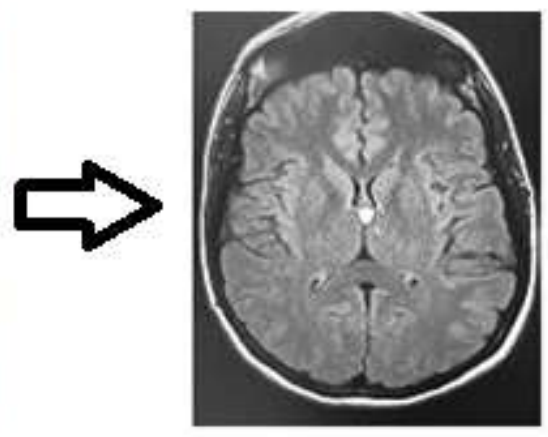

Fig. 3 Grayscale image (8-bit) 


\subsection{MEDIAN FILTERING AND GAUSSIAN HIGH-PASS FILTERING}

MRI and CT Scanners use strong radio waves, magnetic fields, field gradients and radiation. Before automating the process of detecting the Colloid Cyst, it is essential to remove any noise or unnecessary information present in the image that would lead to errors. The preprocessing of the image is required to improve the image quality and extract more delicate details of the image by removing noise and sharpening the image for producing rough edges. An image can be affected by different types of noise. The most common type of noise is the Salt-and-pepper noise that has dark pixels in bright regions and bright pixels in dark regions Median Filters are best known for removing Salt-and-Pepper noise from an image as shown in Figure 5. Median Filters considers each pixel and replaces them by the median in a neighbourhood around the pixel as shown in the below example in Figure 4.
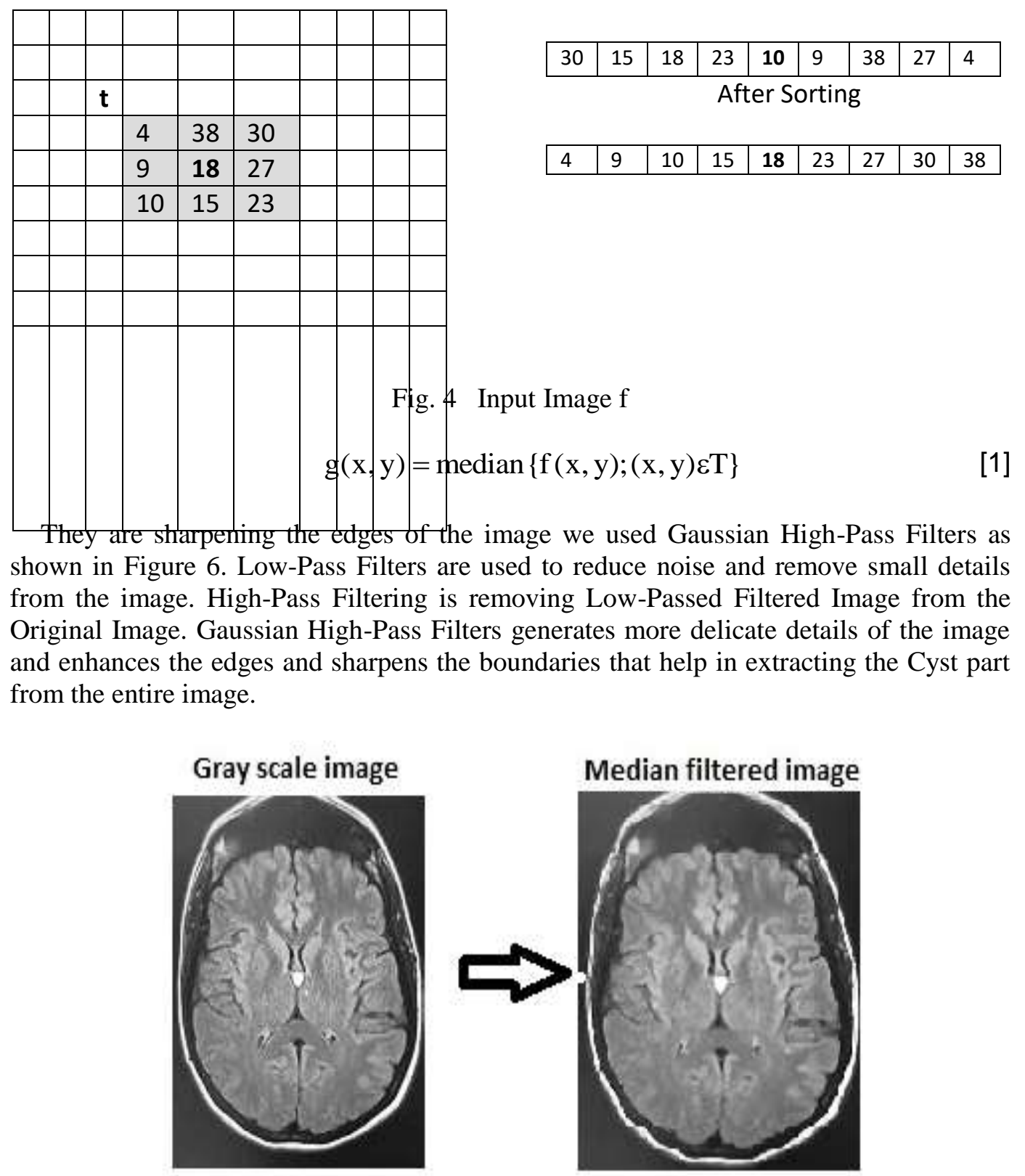

Fig. 5 Applying the Median filter to remove noise 

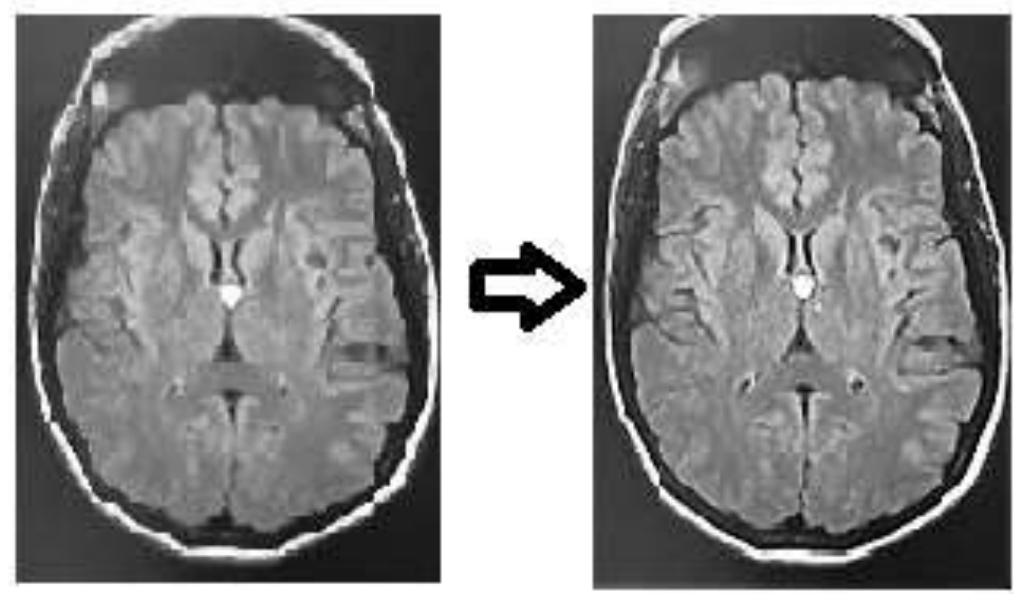

Fig. 6 Applying Gaussian High-Pass filter

\section{COLLOID CYST DETECTION AND ITS APPROXIMATE SIZE}

Segmentation techniques applied to extract the cyst part from the image after the preprocessing techniques. Image Segmentation to identify the cyst manually is very hectic as well as time-consuming, it is vital to automate the segmentation process of identifying the object of interest, its location and boundaries. The primary purpose of Segmentation is to divide an image into regions or small parts that share the same characteristics. Segmentation makes the analysing part easier by extracting meaningful features and identifying the region of interest. There are various methods available for image segmentation. E.g. clustering method, Histogram-based method, Region-growing method, Compression based method etc. Threshold Segmentation is one of the oldest and simplest segmentation methods. In threshold segmentation firstly converts an input grayscale image to binary format. We have used Multilevel Thresholding where the number of thresholds is given in advance, and the best thresholds are found by minimising or maximising a criterion $[11,12]$. Figure 7 shows the implementation of Multilevel Thresholding and Threshold segmentation on the pre-processed image. We have determined multiple thresholds $\left\{\boldsymbol{T}_{1}, \boldsymbol{T}_{2}, \ldots, \boldsymbol{T}_{N}\right\}$ in the histogram of the image $f(\boldsymbol{x}, \boldsymbol{y})$ to segregate the pixels with similar attributes and identify the object of interest in the image[13].

$$
\begin{aligned}
& S(f(x, y))=\left[G_{0} \text { if } f(x, y) \leq T_{1}\right] \\
& S(f(x, y))=\left[G_{1} \text { if } T_{1}<f(x, y) \leq T_{2}\right] \\
& S(f(x, y))=\left[G_{N} \text { if } f(x, y)>T_{N}\right]
\end{aligned}
$$

Where $\mathrm{S}(\boldsymbol{f}(\boldsymbol{x}, \boldsymbol{y}))$ is the Segmented image and $\boldsymbol{G}_{\boldsymbol{L}}$ is the grey-level assigned to the pixel in region $\mathrm{L}$. 

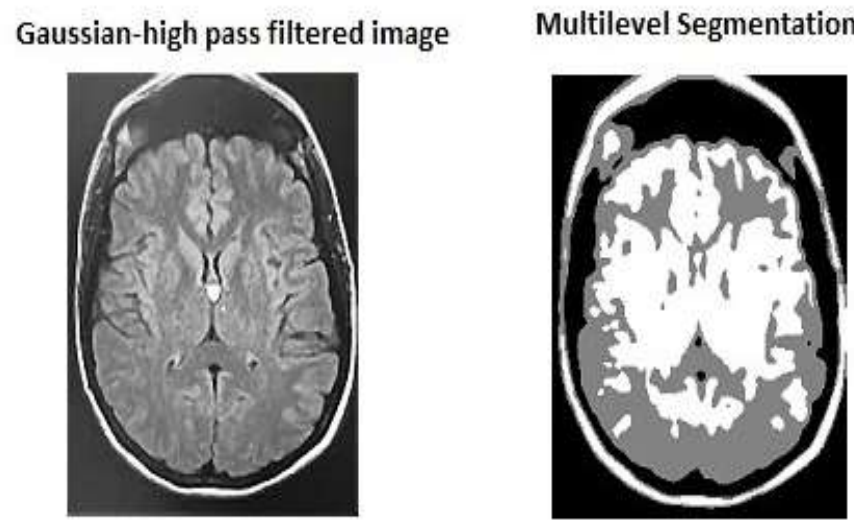

Threshold Segmentation

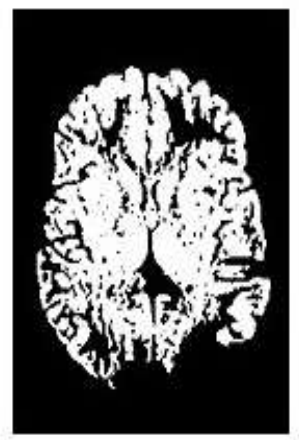

Fig. 7 Multilevel Thresholding and Threshold segmentation

The standard feature of colloid cyst is its location, i.e., the cyst is present in the centre of the brain. In this paper, we have defined the region of interest and cropped the area to detect the presence of a Colloid Cyst. If Colloid Cyst is present, then we have traced the exterior boundaries of the cyst using black and white boundaries (cyst,'no holes') and then plotted the points shown in Figure 8.

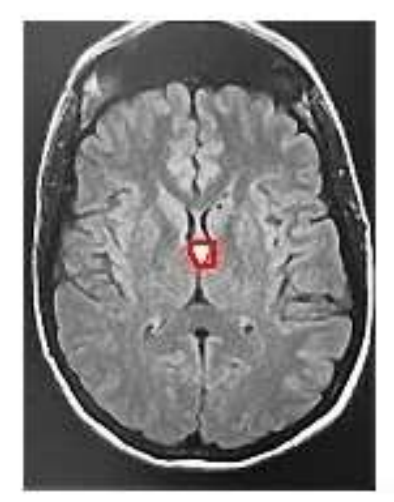

Fig. 8 Identifying the boundaries

Now we have calculated the approximate size of the Colloid Cyst, considering that the image contains only two values, black and white. The maximum image size is taken as 256 x 256 and the binary image represented as

$$
\begin{array}{lll}
\text { Img, BI }=\sum_{\text {WT }} 255 & \sum 255 & {[\mathrm{f}(0)+\mathrm{f}(1)]}
\end{array}
$$

Where $\mathbf{f}(\mathbf{0})$ is a white pixel, $\mathbf{f}(\mathbf{1})$ is a black pixel, WT is width and HT is height. [16]

Pixel $=$ Width $($ WT $) \times$ Height $($ HT $)=256 \times 256$

As we had extracted and marked the Colloid Cyst in white pixels, therefore, no_of_WhitePixel.

$$
\mathrm{W}=\sum_{\mathrm{WT}=0}^{255} \quad \sum^{255} \quad \mathrm{HT}=0 \quad[\mathrm{f}(0)]
$$


We have calculated the approximate size of the Colloid Cyst in $\mathrm{mm}^{2}$.

1 Pixel $=0.264 \mathrm{~mm}$

(Number of White Pixel) $\mathrm{W}=$ width $\mathrm{x}$ height

\section{RESULT ANALYSIS}

The analysis of the current method can be done and verified in various cases with various scenarios. The details of these cases can be explained in detail in the following sections,

Case 1: Figure 10 shows every step that the proposed algorithm follows to detect the presence of a Colloid Cyst from MRI or CT Scanned Images. It is also capable of detecting very small sized Colloid Cyst.
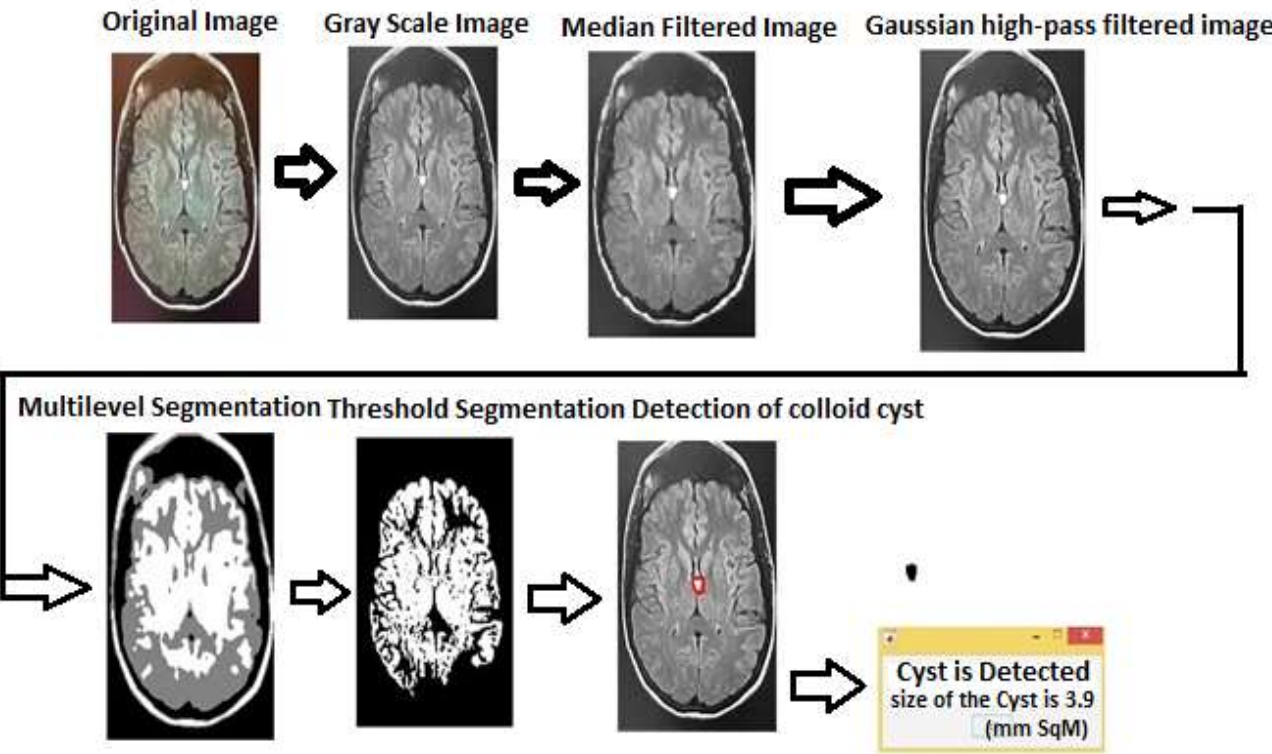

Fig. 9 Full Process of Colloid Cyst Detection

Table I. Analysis of results with different cases.

\begin{tabular}{|c|c|c|c|}
\hline CASE & IMAGE & DESCRIPTION & $\begin{array}{c}\text { DETECTION } \\
\text { TIME }\end{array}$ \\
\hline $\begin{array}{c}\text { Case 1. } \\
\text { Colloid Cyst } \\
\text { detected }\end{array}$ & $\begin{array}{r}\text { Size of Colloid Cyst: } \\
4.9 \mathrm{~mm}^{2}\end{array}$ & $5 \mathrm{~s} 27 \mathrm{~ms}$ \\
& & \\
\hline
\end{tabular}




\begin{tabular}{|c|c|c|}
\hline $\begin{array}{lr}\text { Case } & 1 . \\
\text { Colloid } & \text { Cyst } \\
\text { detected } & \end{array}$ & $\begin{array}{l}\text { Size of Colloid Cyst: } \\
5.12 \mathrm{~mm}^{2}\end{array}$ & $4 \mathrm{~s} 8 \mathrm{~ms}$ \\
\hline $\begin{array}{lr}\text { Case } & 1 . \\
\text { Colloid } & \text { Cyst } \\
\text { detected } & \end{array}$ & $\begin{array}{l}\text { Size of Colloid Cyst: } \\
2.31 \mathrm{~mm}^{2}\end{array}$ & $5 \mathrm{~s} 25 \mathrm{~ms}$ \\
\hline $\begin{array}{l}\text { Case 2. The } \\
\text { absence of a } \\
\text { Colloid Cyst }\end{array}$ & $\begin{array}{l}\text { No Abnormalities } \\
\text { found }\end{array}$ & $9 \mathrm{~s}$ \\
\hline $\begin{array}{l}\text { Case 3. The } \\
\text { absence of a } \\
\text { Colloid Cyst } \\
\text { Presence of } \\
\text { other Cyst }\end{array}$ & $\begin{array}{l}\text { Presence of cyst, but } \\
\text { according to the } \\
\text { location it is not a } \\
\text { Colloid Cyst }\end{array}$ & $8 \mathrm{~s} 14 \mathrm{~ms}$ \\
\hline
\end{tabular}

Considering the high risk of sudden death due to Colloid Cyst, we effectively use Image processing techniques in MATLAB for the automation of detecting Colloid cyst process in Brain from MRI or CT scanned images. In Table I, we have analysed the results of our proposed algorithm to demonstrate what sets it aside from other existing algorithms and to measure its effectiveness and accuracy by testing different cases. As we can see in Table I, the proposed algorithm takes a minimal amount of time to detect the Colloid Cyst, irrespective of the size of the image. Average time taken is between $5 \mathrm{sec}$ to $12 \mathrm{sec}$, and the accuracy rate is $98 \%$. It can detect tiny size Colloid Cyst as well. It extracts and marks the exact shape of the cyst, therefore making the calculation for the size of the Colloid Cyst almost accurate. 


\section{CONCLUSION}

The proposed methodology accurately detects and simplifies the process of detecting the presence or absence of a Colloid Cyst in Brain from MRI or CT Scanned images, through MATLAB. The proposed algorithm provides three cases to find a cyst in the brain. Firstly, if a Colloid Cyst is detected, it displays the approximate size of the cyst. Secondly, if there is an absence of any abnormalities, it displays the original image mentioning that "No Colloid cyst present". Thirdly if there is a Cyst present but not a Colloid cyst, the algorithm would mark the exterior boundaries of the cyst, but according to the location, it displays that it is not a Colloid Cyst. This helps the diagnosis of Colloid Cyst more natural way with accuracy and less time consuming for doctors and radiologists. In future, we would like to extend this work and develop algorithms to detect every type of Cyst in Brain depending upon its location as well as other identification criteria and mention the type of cyst along with its size and further vital details required for faultless treatment.

\section{REFERENCES}

[1] Al-Sharydha A M et. al., "A unique finding of cavum velum interpositum colloid-like cyst and literature review of a common place lesion in an uncommon place", International Journal of General Medicine, Vol.11, 2018, Pp: 301-305.

[2] Alexander C. Mamourian et. al., "Colloid Cyst of the Third Ventricle: Sometimes More Conspicuous on CT than MR", AJNR Am J Neuroradiol, 19, May 1998, pp. 875-878.

[3] Jeremy P. Morgan et. al., "Treatment of colloid cyst of the third ventricle by stereotactic aspiration followed by radiosurgery: Report of four cases", International Journal of Neurosurgery and Neuroscience, Vol.9, No.3, January 2018.

[4] Karishma Sheikh et. al.,"Clustering-based Segmentation Approach to Detect Brain Tumor from MRI Scan", International Journal of Computer Applications (0975 - 8887), Vol. 118, No. 8, May 2015, pp. 36-39.

[5] Ed-EdilyMohd. Azhari, et. al., "Brain Tumor Detection and Localization in Magnetic Resonance Imaging", International Journal of Information Technology Convergence and Services (IJITCS), Vol.4, No.1, February 2014, pp. 1-11.

[6] Vrushali D. Dharmale et. al., "Segmentation and Canny Edge Method in MRI Brain Cyst Detection", International Journal of Advanced Computer Research (ISSN (print): 2249-7277 ISSN (online): 22777970), Vol. 3, No. 4, Issue 13, December-2013, pp.289-293.

[7] Lalit P. Bhaiya et. al., "Classification of MRI Brain Images using Neuro-Fuzzy Model", International Journal of Engineering Inventions ISSN: 2278-7461, Vol. 1, Issue 4, September 2012, pp. 27-31.

[8] Mashal Tariq et. al., "Image Processing with the specific focus on early tumour detection", International Journal of Machine Learning and Computing, Vol. 3, No. 5, October 2013, pp. 404-407.

[9] Qaiser Javed, Arpan Dutta, "Third Ventricular Colloid Cyst and Organic Hypomania", Progress in Neurology and Psychiatry, November/December 2014, pp. 18.

[10] https://diagnosticpathology.biomedcentral.com/articles/10.1186/1746-1596-7-144[Last accessed on 02.08.2018]

[11] http://www.ncbi.nlm.nih.gov/pmc/articles/PMC1550234. [Last accessed on 08.08.2018]

[12] http://mstrzel.eletel.p.lodz.pl/mstrzel/pattern_rec/filtering.pdf[Last Accessed on 09.08.2018]

[13] http://www.mecs-press.org/ijisa/ijisa-v5-n11/IJISA-V5-N11-3.pdf[Last accessed on 10.08.2018]

[14] http://www.medicalnewstoday.com/articles/181727.php [Last accessed on 07.12.2017]

[15] http://www.abta.org/secure/resource-one-sheets/cysts.pdf [Last accessed on 13.08.2018] 